\title{
Hybrid Adaptive Call Admission Control Mechanism for Ensuring QoS in 3GPP: LTE Networks
}

\author{
J Vijay Franklin \\ Assistant Professor Senior Grade, Department \\ of Computer Science \& Engineering \\ Bannari Amman Institute of Technology, \\ Sathyamangalam, Tamilnadu
}

\author{
K Paramasivam \\ Professor Department of Electronics \& \\ Communication Engineering \\ Bannari Amman Institute of Technology, \\ Sathyamangalam Tamilnadu,India
}

\begin{abstract}
Third Generation Partnership Project (3GPP) Long Term Evolution (LTE) system is targeted to efficiently guarantee the quality of service (QoS) of services such as audio/video streaming, gaming and Voice over IP (VoIP). So the system resource allocation should obtain the multiuser diversity gain making full use of the channel condition, and take the quality of service (QoS) of different services into account. Due to the heavy user's space and limited Resource Block (RBs), it is infeasible to guarantee all ongoing users' QoS. Then, some call are blocked and lost. The proposed method reduces the handoff blocking probability in LTE wireless networks. Hybrid Adaptive call admission control scheme performs the QoS operation based on the priority at the time of call admission. This method reduces new call blocking probability.
\end{abstract}

\section{General Terms}

Call Admission Control, Channel allocation schemes, Call Blocking rate.

\section{Keywords}

RSS, QoS, HCAC algorithm, Handoff

\section{INTRODUCTION}

$3^{\text {rd }}$ Generation $(3 \mathrm{G})$ mobile telecommunications is a generation of standards for Mobile phones and mobile telecommunication services satisfies the International Mobile Telecommunications-2000(IMT-2000) specifications by the International Telecommunication Union. [2]Application services are wide-area wireless voice telephone, mobile Internet access, video calls and mobile TV, all in a mobile environment. Deployment of third-generation or 3G wireless systems based on Wideband Code-Division Multiple Access (WCDMA) and Code Division Multiple Access (CDMA) 2000 wireless technologies is now gaining momentum around the world. [9]An evolution of $3 \mathrm{G}$ wireless technologies has already started by Third Generation Partnership Project (3GPP) through the introduction of high-speed downlink packet access (HSDPA) and enhanced uplink. These technologies provide $3 \mathrm{GPP}$ with a radio access technology that will be highly competitive in the mid-term future.

3GPP Long Term Evolution (LTE) is a high-speed data transmission standard based on GSM and UMTS/HSPA network technologies. The standard is maintained as a project of the $3 \mathrm{GPP}$, the European Telecommunications
Standards Institute (ETSI). The goal of LTE is to increase the capacity and speed of wireless data networks utilizing cutting-edge hardware and Digital Signal Processing

(DSP) techniques that have recently been developed. Its wireless interface is incompatible with $2 \mathrm{G}$ and $3 \mathrm{G}$ networks, and so it must be operated on separate wireless spectrum. Features of LTE include an all-IP flat network architecture, end-to-end QoS including provisions for lowlatency communications, peak download rates nearing 300 Mbps and upload rates of 75 Mbps, capacity exceeding 200 active users per cell, the ability to manage fast-moving mobiles, and support for multi-cast and broadcast streams.LTE system is targeted to efficiently guarantee the Quality of Service (QoS) of services such as audio/video streaming, gaming and voice over IP (VoIP). So the system resource allocation should obtain the multi-user diversity gain making full use of the channel condition, and take the QoS of different services into account. However, because of too much users and limited Resource Blocks (RB), it is infeasible to guarantee all ongoing users' QoS. Then, some call are blocked and lost. Two types of call blocking are: New-call blocking and Handoff call dropping. The first type refers to the failure of the initial call connection establishment, whereas the second type refers to the blocking of in-service calls when they move from one cell to another. From the users' point of view, it is more frustrating to lose a call that has already begun than to be prevented from establishing a new call.

\section{RELATED WORK}

\section{LOOKING AROUND (LA) ALGORITHM}

Earlier algorithms for call admission control are based on the effective traffic load for the target cell if one call is accepted. These algorithms ignore the interference effect of the to-be-accepted call on the neighboring cells. Yue Ma et al., (2000) decide Call admission based on the effective traffic loads for both the target cell and the neighboring cells. In addition, to prioritize handoff calls, the idea of soft guard channel, which reserves some traffic load exclusively for handoff calls, is also used. [1]This algorithm is denoted as LA algorithm as the call is made after 'looking around' at the neighborhood cells. The call is accepted if the maximum effective load for the adjacent cells is below the threshold.

[12]The drawback of the conventional methods is that they cause the increase of the dropped calls in the adjacent 
cells. Consequently, the blocking probability in the LA algorithm is increased. Because of the capacity of the CDMA system is interference limited, when a new call is coming, the admission decision is based on the loads of both the local and the adjacent cells. The conventional CAC methods only consider the local effect. Therefore their blocking probabilities are lower than the ones obtained from LA algorithm.

\section{ADMISSION CONTROL (AC) ALGORITHM}

To maintain the QoS of in-progress sessions in a cell it is important to admit a new radio bearer only if all the new bearer and the existing sessions can be guaranteed QoS according to their requirements. M. Anas et al., (2008) propose an AC algorithm for LTE uplink utilizing the fractional power control formula agreed in 3GPP which provisions QoS. AC algorithm modifies itself with respect to load conditions variations without additional complexity. AC algorithm checks if the current resource allocation can be modified so as to admit the new user and satisfy the Guaranteed Bit Rate (GBR) requirements of all the active users and the new user. In this algorithm the closed loop adjustments of fractional power control are not taken into account.

[4]The admission criterion for the new user is that the sum of the required number of Physical Resource Blocks (PRBs) per Time Transmission Interval (TTI) by a new user requesting admission and existing users is less than or equal to the threshold which is the total number of PRBs in the system bandwidth. In these schemes, the authors do not distinguish between the handoff call and the new call. Then, the authors do not take the prioritization between the call while basing the type of call and their QoS requirement. Also, the management of threshold is static; it does not depend on the type of call.

\section{CAC ALGORITHMS}

\section{Connection Access Control Scheme}

Haipeng LEI et al., (2008) propose a resource allocation algorithm and a Connection Access Control scheme for LTE systems with heterogeneous services. The CAC scheme introduces a transmission guard interval which gives high priority to real-time (RT) service packets approaching the delay deadline. The Connection Admission Control scheme can adaptively adjust the threshold according to the network condition. It balances the ongoing connections of different classes of traffics but also be easy to reserve resource and support handover users potentially.

This scheme for LTE systems with heterogeneous services introduces a transmission guard interval which gives high priority to the real time (RT) service packets approaching the delay deadline. In this approach, the authors treat all the calls Handover Call and New Call (HC \& NC) in the same way.

\section{Channel Allocation Scheme}

The channels of each cell can be divided into two parts for;

- handoff call
- new call.

Ojesanmi O.A et al., (2009) proposed an adaptive threshold channel allocation scheme for new and handoff calls in wireless multimedia network. To give handoff call higher priority over new calls, the handoff call is allowed to preempt the new call when it finds no channel available on its arrival. The interrupted new call goes to the buffer until channel is available at the new call reserve channels. The call in the buffer is served using ticket scheduling[6].

The guard channel scheme (GCS) is to reserve a fixed percentage of the system's capacity for handoff calls. [5] The advantage of this scheme lies in the simplicity of deployment, since there is no need to exchange control information between the base stations. However, with a small portion of handoff call, GCS results not only in increased blocking probability of new call, but also in inefficient resource utilization, because only a few handoff calls are able to use the reserved channels exclusively. On the other hand, with a large portion of handoff calls it is difficult to guarantee the service requirements of handoff call.

\section{QoS - CAC ALGORITHM}

[3] In QoS-CAC algorithm admits all connections (handoff and new calls) with QoS guaranteeing within the network. The new connection requesting for the establishment, that are classified based on the associated services. Qos-CAC performs the operations based on the service requests like RTPS and NRTPS queues. it provides highest priority to UGS connections requests followed by RTPS and NRTPS connection requests. The new calls that are arrived into a network will be classified into UGS,RTPS and NRTPS connections. The highest priority has given for the UGS connection that deals with fixed size packets, and QoSCAC performs the RTPS Queue with the variable sized packets. The least priority is given for the NRTPS services that deals with non real time with variable sized packets

Balancing of traffic load among multiple cells in wireless network allows for a better utilization of the radio resources has to be performed. The LTE utilizes a network-controlled and the User Equipment (UE) assisted handover procedure for mobility in connected mode. UE measures the power of signal strength RSS and sends the measurement report to the serving eNodeB. Since the RSS of an UE is related to the distance between an UE and its associated eNodeB, the RSS value at the time $t$ is given by:

$$
R S S[t]=P_{t r}-10 \log (d)+X_{d B} \ldots \ldots \ldots \ldots \ldots \ldots(1)
$$

Where $\mathbf{d}$ :is the distance between the UE and the associated. AP,

$\mathbf{P}_{\text {tr }}:$ is the transmitted signal power, and

$\mathbf{X}_{\mathbf{d B}}$ : is a Gaussian random variable with zero mean

The eNodeBs candidates are those which relate to significant RSS higher than a threshold value RSS $_{\text {Add }}$ :

$$
\mathbf{R S S}_{\mathbf{i}}>\mathbf{R S S}_{\text {Add }}
$$

The serving eNodeB then makes the decision of handover based on the received measurement reports. The necessary condition of the handover decision is that the 
value of the RSS relating to the serving eNodeB is degraded (i.e. reached a limiting value threshold RSS $_{\text {Drop }}$ ). Thus the necessary condition of handover decision is checked by the following condition:

$$
\mathbf{R S S}_{\text {serv }}<\mathbf{R S S}_{\text {Drop }}
$$

The serving eNodeB carries out a cell selection process consists in allocating the user to the cell with the lowest load level and which is provided that it can fulfill the QoS requirements requested by the UE. In turn, an admission control may be performed by the selected target eNodeB according to the received quality of service information. In case that there is no capacity available for the handoff call in the selected cell (i.e. the admission control is not passed), another cell from the candidates cells will be selected instead. Once the decision of the handover is taken, the serving eNodeB informs the UE by the new eNodeB and orders to him to ask the detachment and to achieve the handover. The target eNodeB can now start sending data to the UE and, at the same time, send a path switch message to the Serving Gateway to inform that the UE has changed cell.

\section{CONNECTION ADMISSION CONTROL AND RBS RESERVATION ALGORITHM}

A CAC algorithm is considered that takes into account the separation between incoming traffic for each class and prioritizes handoff calls over new calls. three class of service of arrival calls of each class depending on their QoS profile, such as latency tolerance.

- $\quad$ non real time service (NRT),

- real time tolerant service (RT-TLR) and

- real time intolerant service (RT-INTLR).

On the arrival of calls with similar type (i.e. HC or NC) to an overloaded cell (i.e. the number of resource blocks available is insufficient to satisfy the requests for connections), the latter will await the acceptance by storing them in specific queues. These calls will be treated differently since their latency depends on the type of traffic. Thus, three different queues are used (for each class of service) for each type of call.

The latency $T_{A, i}$ of a user requiring a request depends only on emission $\mathrm{T}_{\mathrm{e}, \mathrm{i}}$ and the reception time $\mathrm{T}_{\mathrm{r}, \mathrm{i}}$ of the request:

$$
\mathbf{T}_{\mathrm{A}, \mathrm{i}=} \mathbf{T}_{\mathrm{r}, \mathrm{i}}-\mathbf{T}_{\mathrm{e}, \mathrm{I}}
$$

The requests in the wait state will be treated while being based on the latency criteria. In other words, the requests which have the minimum tolerated latency and provided that it does not exceed the maximum latency delay will be treated the first. If one has two requests for $\mathrm{HC}$ (or NC) waiting in two different files (i.e. they ask for different applications of class of service) and checking the temporal constraints:

- A request for $\mathrm{HC}$ (or NC) with class of service

$$
\mathrm{i}: \mathbf{T}_{\mathbf{A i}}<\mathbf{D}_{\text {max }, \mathbf{i}}
$$

RT : real time system

NRT : Non Real time system

TLR : real time tolerance
- A request for $\mathrm{HC}$ (or $\mathrm{NC}$ ) with class of service $\mathrm{j}$ :

$$
\mathbf{T}_{\mathrm{Aj}}<\mathbf{D}_{\text {max }, \mathbf{j}}
$$

The HC (or NC) which will be treated the first is that which solves the following equation:

$$
t_{r}=\min \left(D_{\max , i^{-}} \mathbf{T}_{A i}, D_{\text {max }, j} \mathbf{T}_{A j}\right)
$$

To satisfy the prioritization for the handover call over the new call taking the QoS requirement, the CAC proposes a RBs reservation algorithm. According to the service contract and to the decision of CAC, the eNodeB will execute the RB reservation algorithm. Thus, the algorithm will be triggered whenever there is a call arrival acceptance event or a service departure event. Ideally, every call in a cell should be allocated the maximum number of RBs (RBmax) whenever possible. However, if the cell is over-loaded, some of the calls in the cell might receive a number of RBs lower than the requested RB.

Thus, for the acceptance of a $\mathrm{HC}$ request, a degradation procedure is triggered to degrade the number of allocated RBs of some ongoing calls in the cell to attempt to allocate required RB (Rbreq) as follows. Calls with the largest allocated bandwidth greater than Rbmin and the lowest priority (i.e. carrying a non real time service) are degraded to have lower number of RBs not less than Rbmin. If one arrives at a level of degradation in which is found that the sum of the released RBs is higher than those required by the $\mathrm{HC}$ then this last will be accepted and will be allocated the requested resources blocks. If all above tests fail, then the dropping of the handoff call is done. But, to treat the requests for new connection on standby if the cell is overloaded, in the first time the call which does not exceed its maximum latency and which tolerates the minimum of remaining latency is selected. To accept this call, some calls in the course of communication which carrying applications with non real time service, its resources allocated blocks will be degraded up to the required level (Rbreq) to accommodate this NC. If all above tests is not complied with, then blocking of arrival call is done else the $\mathrm{NC}$ will be accepted and it will allocate their required RBs.

As a call leaves the cell, whether outgoing handoff call or a call completion, the total available bandwidth increases. The RBs algorithm uses different of above ideas to allocate, increase, and decrease the number of RBs for the calls in a cell.

\section{HYBRID CAC ALGORITHM}

Hybrid CAC(HCAC) algorithm used here uses the resource block strategy to allocate the resources based on the call type i.e. new call or Handoff call. The parameters used are

$\mathrm{RB}_{\max } \quad$ : maximum number of $\mathrm{RBs}$ required

$\mathrm{RB}_{\text {min }} \quad$ : minimum number of $\mathrm{RBs}$ required

$\mathrm{RB}_{\text {req }} \quad$ : number of required $\mathrm{RBs}$

$\mathrm{D}_{\max , \mathrm{i}} \quad$ : tolerable maximum delay

$\mathrm{T}_{\mathrm{A}} \quad$ : latency 
INTLR : real time intolerance

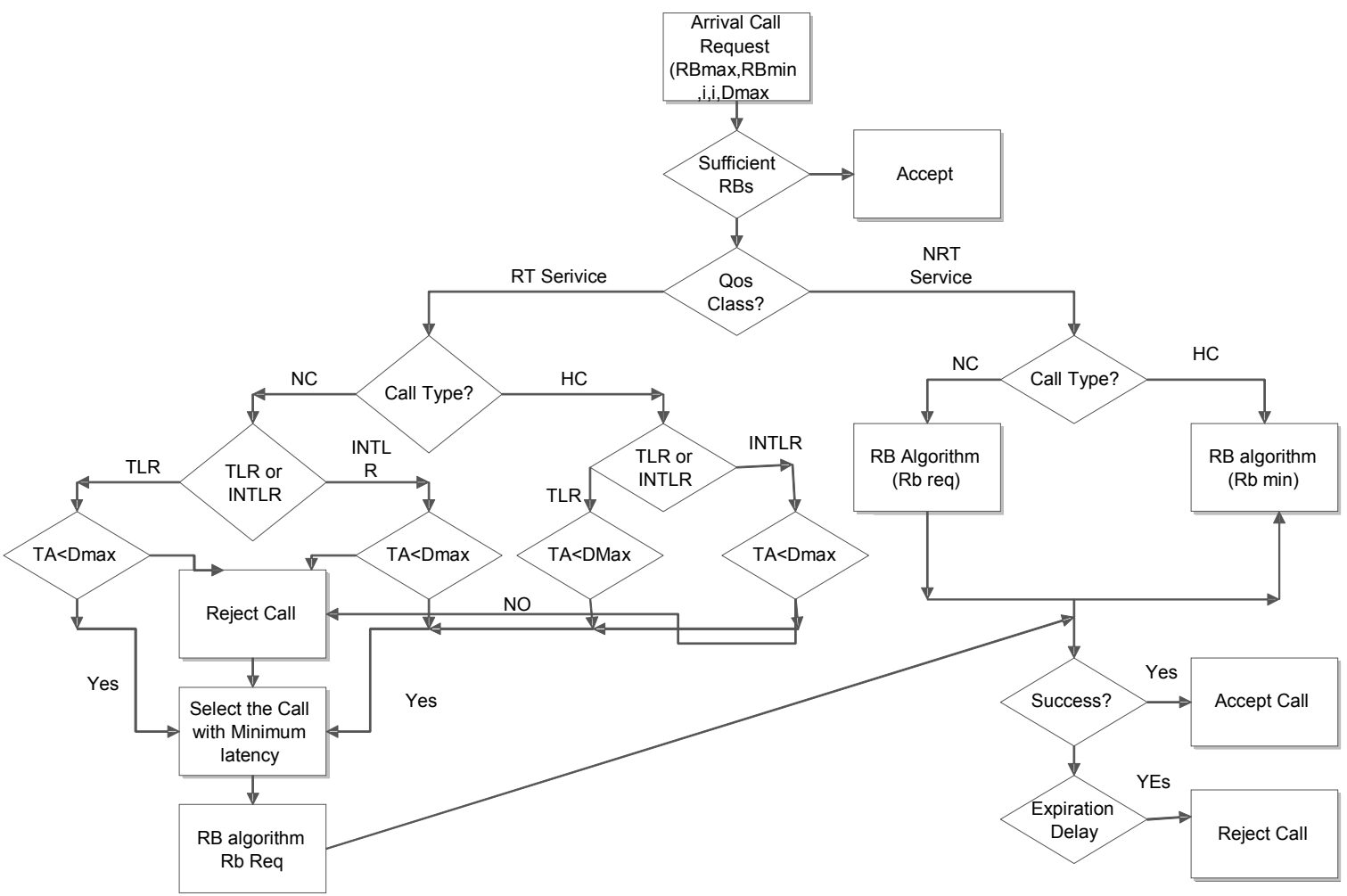

Figure: HCAC algorithm

The algorithm is described as follows

- When a call arrives the required parameters like $\mathrm{RB}_{\text {max }, \mathrm{i}} \mathrm{RB}_{\text {req }, \mathrm{i}} \mathrm{RB}_{\text {min,i }} \mathrm{D}_{\text {max }, \mathrm{i}}$ are checked.

- If the resources are sufficient then the call is accepted else the type of service is determined.

- The QoS call and call type is determined. For $\mathrm{NC} \mathrm{RB}_{\text {req }}$ parameter is used and for $\mathrm{HC} \mathrm{RB}_{\text {min }}$ parameter is used.

- If the service type is RT then the call type is determined. For both the condition $\mathrm{TA}<\mathrm{Dmax}$ is checked if true then proceeded to next step, else the call is rejected.

- If the condition is satisfied then the call with minimum latency is checked and resource block algorithm is used.

- If sufficient RBs are present then call is accepted else expiration delay is introduced. At the expiration of the delay if still the resources are not available then call is rejected.

- If the service type is NRT then call type is determined and RB algorithm is used. If the resources are available the call is accepted else expiration delay is introduced.

- At the expiration of the delay if still the resources are not available then call is rejected. The figure following figure shows the flow chart for HCAC algorithm.

\section{IMPLEMENTATION OF THE SYSTEM}

The system implementation requires visual studio 2008 and Matlab R2010a as they support simulink required for LTE simulation.

The steps for the implementation are as follows

- The total numbers of users are determined.

- The percentage of total request that will go for each network are determined.

- The arrival rate and departure rate is determined based on which the throughput is calculated.

- The blocking probability is calculated.

- The figure 1,2,3 shows cost incurred for the number of users in network to a specified throughput of that network. The graph shows that for high average percentage of user the cost is also high.

- The figure 4 shows the cost for the corresponding networks and the cost for the combined result.

- The figure 5 call blocking $\operatorname{rate}(\mathrm{CBR})$ for the average percentage of user in a network. The graph shows that as the number of user increases the CBR also increases.

- The figure 6 shows blocking probability for traffic load in corresponding networks. The graph shows that as traffic load decreases so does the blocking probability. 


\section{INPUT:}

No. of users $(1-1000)=100$

Percentage of total request that will go to Network- $1=10$ Percentage of total request that will go to Network- $2=50$ Percentage of total request that will go to Network-3 $=100$ Row APUSR

CBR $\rightarrow$ Throughput of a network. $\rightarrow$ Average Percentage of User MUSE-VDA

$\rightarrow$ Call Blocking Rate

$\rightarrow$ Blocking Probability

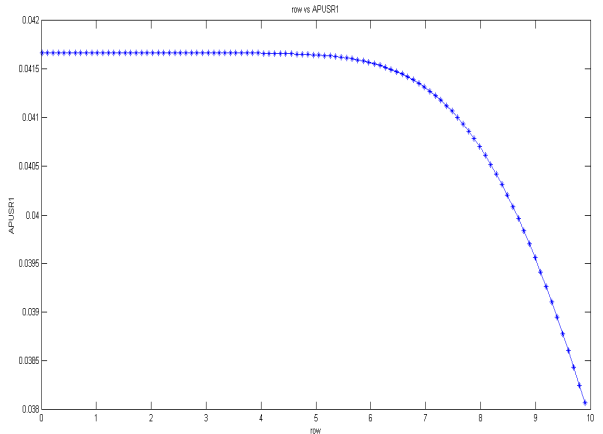

Figure 1 Row vs APUSR1

Figure 1 shows cost incurred for the number of users in network1 to a specified throughput of that network. The graph shows that for high average percentage of user the cost is also high.

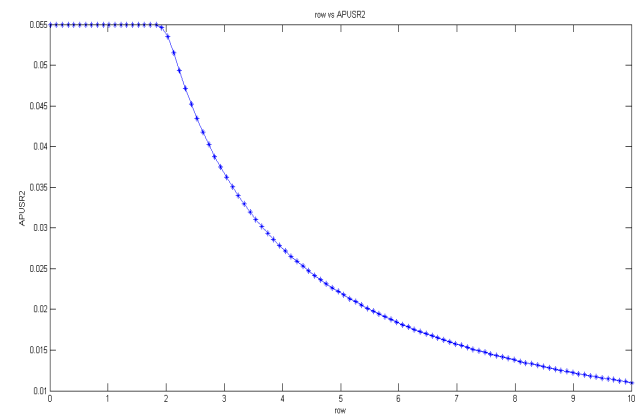

Figure 2 Row vs APUSR2

Figure 2 shows cost incurred for the number of users in network2 to a specified throughput of that network. The graph shows that for high average percentage of user the cost is also high.

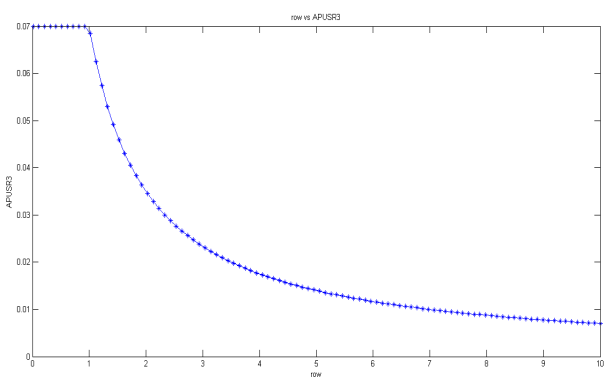

Figure 3 Row vs APUSR3

Figure 3 shows cost incurred for the number of users in network 3 to a specified throughput of network.

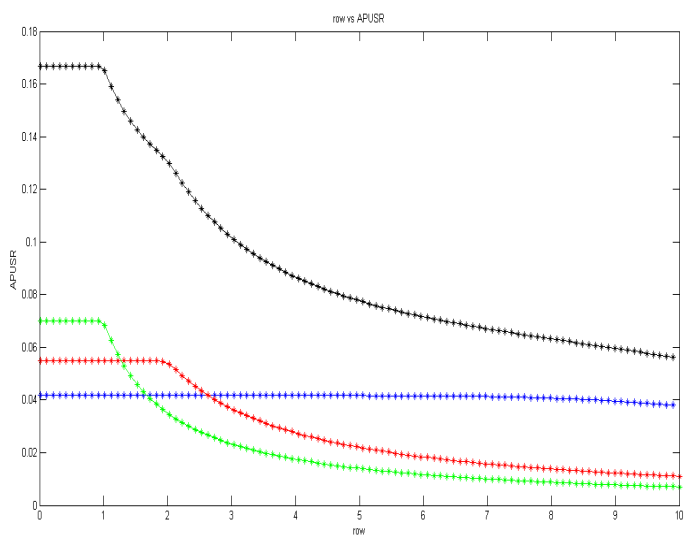

Figure 4 Row vs APUSR

Figure 4 shows the cost for the corresponding networks and a combined result.

RED $\rightarrow$ network1

GREEN $\rightarrow$ network2

BLUE $\rightarrow$ networks3

BLACK $\rightarrow$ combined cost of average percentage

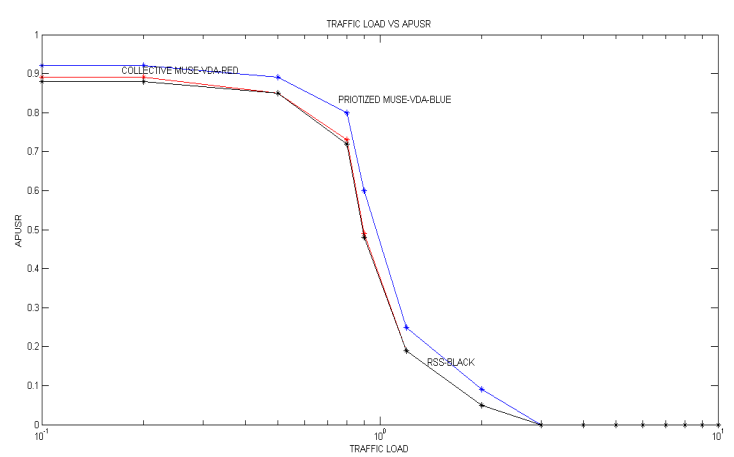

Figure 5 CBR vs APUSR

Figure 5 shows call blocking rate(CBR) for the average percentage of user in a network. The graph shows that as the number of user increases the CBR also increases.

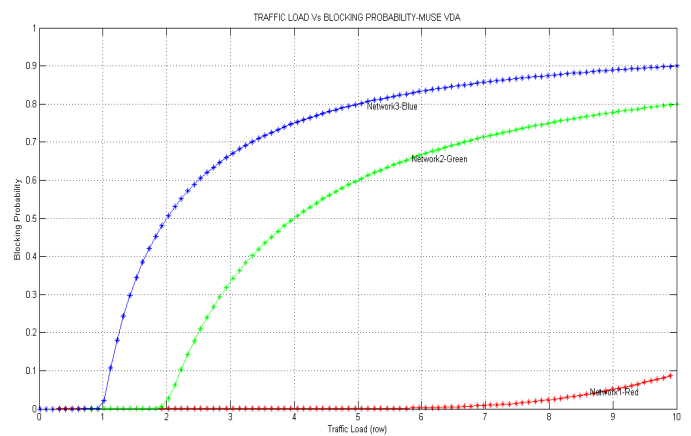

Figure 6 Traffic Load vs. Blocking Probability-MUSE VDA

Figure 6 shows blocking probability for traffic 
load in corresponding networks. The graph shows that as traffic load decreases so does the blocking probability.

\section{CONCLUSION}

During the handoff in LTE networks it is very important to provide resources such that the call is continued. The handoff call should given more importance than the new call as it more frustrating to lose an ongoing call than a new call. Thus during the handoff the call dropping should be reduced. The HCAC algorithm described uses resource allocation scheme to guarantee the QoS. This Resource Block reservation can achieve an improvement on the call blocking probability and keeping inefficient resource utilization. The results have shown that as the number of user increase the number of new call blocking increases. The expiration delay provided enables the user to wait for allocation of resources instead of disconnecting the call immediately. This system has the advantages of efficient handover call continuation. The scope of this concept can to extended to inter LTE environment. The existing methodology provides a higher rate new call blocking than the handover call but the system is time consuming. Hence the proposed methodology will be focused on more efficient resource utilization, to reduce the time complexity and to further improve the QoS.

\section{REFERENCES}

[1] Khitem Ben Ali, Faouzi Zarai, Lotfi Kamoun (2010), "Reducing handoff dropping probability in $3 \mathrm{GPP}$ LTE Network" IEEE.

[2] Yue Ma, James J. Han, Kishor S. Trivedi(2000), “Call Admission Control for Reducing Dropped Calls in Code Division Multiple Access (CDMA) Cellular Systems", IEEE INFOCOM 2000.

[3] M. Anas, C. Rosa, F. D. Calabrese, P. H. Michaelsen, K. I. Pedersen, and P. E. Mogensen (2008), "QoS-Aware Single Cell Admission Control for UTRAN LTE Uplink", VTC Spring,IEEE.

[4] Haipeng LEI, Mingliang YU, Anjun ZHAO, Yongyu CHANG, and Dacheng YANG, (2008), "Adaptive Connection Admission Control Algorithm for LTE Systems", VTC Spring, IEEE.
[5] Oyebisi T.O, Ojesanmi O.A,(2008) "Development of congestion control scheme for wireless mobile network", Journal of Theoretical and Applied Information Technology.

[6] Ojesanmi O.A and Famutimi R.F,(2009), "Adaptive threshold based channel allocation scheme for multimedia network", IJCSNS International Journal of Computer Science and Network Security, VOL.9 No.1.

[7] M. Sanabani, S. Shamala, M. Othman and 1. Desa,(2006), "Adaptive call admission control for prioritized adaptive services in wireless/mobile multimedia cellular networks", IJCSNS International Journal of Computer Science and Network Security, VOL.6 No.38.

[8] Nasıf Ekiz, Tara Salih, Sibel Küçüköner, and Kemal Fidanboylu(2006), "An Overview of Handoff Techniques in Cellular Networks", World Academy of Science, Engineering and Technology.

[9] $3^{\text {rd }}$ Generation Partnership Project,(2008) “Technical Specification Group Radio Access Network; Evolved Universal Terrestrial Radio Access (EUTRA) and Evolved Universal Terrestrial Radio Access Network (E-UTRAN) V8.5.0", TS36.300.

[10] Kyungmin Kim, Hyunduk Jung, Jaiyong Lee(2009), "A Seamless Voice Call Handover Scheme for Next Generation Cellular Network", Proceedings of the $15^{\text {th }}$ Asia-Pacific Conference on Communications (APCC 2009)-186.

[11] Ojesanmi O. A, Ojesanmi A. and Makinde O.(2009), "Development of Prioritized Handoff Scheme for Congestion Control in Multimedia Wireless Network", Proceedings of the World Congress on Engineering 2009

[12] Yuguang Fang, and Yi Zhang, (2002), "Call Admission Control Schemes and Performance Analysis in Wireless Mobile Networks", IEEE Transactions On Vehicular Technology, VOL. 51, NO. 2. 